\title{
On the Diameter of Compact Homogeneous Riemannian Manifolds
}

\author{
By \\ Kunio Sugahara*
}

\section{Introduction}

Let $M$ be a compact Riemannian manifold. The diameter $d(M)$ of $M$ is defined to be the maximum of $d(p, q) p, q \in M$, where $d($, ) denotes the distance function on $M$ induced by the Riemannian metric.

The main purpose of this paper is to find a positive consiant $d$ such that the diameter $d(M) \geqq d$ when the sectional curvature $K \leqq 1$.

In this paper we consider the case that the manifold $M$ is homogeneous. In [3] the author proved that $d=\pi / 2$ if the manifold has a big isotropy subgroup. It has been left to study the case that the isotropy subgroup is finite. Hence we shall mainly study invariant metrics on a Lie group and prove that the number $d>0.23$ if the sectional curvalure $K \not \equiv 0$ (Theorem 5.1).

\section{$\S 1$. Fixed Points of Isometries}

Let $M$ be a compact $C^{\infty}$ manifold with a Riemannian metric $g$. Let $d_{g}($, denote the distance function on $M$ induced by $g$. Let $I(M, g)$ denote the group of isometries of $(M, g)$. Let $p$ be a point of $M$. We denote by $I_{p}(M, g)$ the isotropy subgroup of $I(M, g)$, i.e., $I_{p}(M, g)=\{a \in I(M, g) ; a p=p\}$. Let $A$ be a connected subgroup of $I_{p}(M, g)$. Put $F(A)=\{x \in M ; A x=x\}$. Then it is easy to see that $F(A)$ is a disjoint union of closed totally geodesic submanifolds of $M$. For a curve $c:[0,1] \rightarrow M$, we denote by length $(c)$ the length of $c$ with respect to the metric $g$.

Lemma 1.1. Let $A$ be a connected subgroup of $I_{p}(M, g)$ with $\operatorname{dim} A \geqq 1$.

Received March 1, 1979.

* Research Institute for Mathematical Sciences, Kyoto University.

Present address: Department of Mathematics, Osaka Kyoiku University, Osaka 543, Japan. 
Then $F(A) \varsubsetneqq M$. Let $\gamma:[0,1] \rightarrow M$ be a geodesic starting from a point of $F(A)$ in the normal direction to $F(A)$. Assume that the sectional curvature $K_{g} \leqq k$ $(k>0)$ and length $(\gamma) \leqq \pi / 2 \sqrt{k}$. Then $d_{g}(F(A), \gamma(1))=\operatorname{length}_{g}(\gamma)$, i.e., the injectivity radius of $F(A)$ is not less than $\pi / 2 \sqrt{k}$.

Proof. In case that $k=1$ and $A$ is the identity component of $I_{p}(M, g)$, this is Proposition 4.2 in [3]. The proof in it is still valid for the case that $A$ is a connected subgroup of $I_{p}(M, g)$ without any change. Hence we obtain

$$
\begin{aligned}
\text { length }_{g}(\gamma) & =\frac{1}{\sqrt{k}} \text { length }_{k g}(\gamma) \\
& =\frac{1}{\sqrt{k}} d_{k g}(F(A), \gamma(1)) \\
& =d_{g}(F(A), \gamma(1)),
\end{aligned}
$$

since $K_{k g}=K_{g} / k \leqq 1$ and length ${ }_{k g}(\gamma)=\sqrt{k}$ length $_{g}(\gamma) \leqq \pi / 2$.

Q.E.D.

\section{§2. The Length of a Killing Vector Field}

Let $(M, g)$ be a compact Riemannian manifold as in Section 1 .

Theorem 2.1. Let $\xi$ be a non-trivial Killing vector field on the Riemannian manifold $(M, g)$. Put $\alpha=\max _{x \in M} g(\xi, \xi)_{x}$, and $\mathscr{F}=\left\{x \in M ; g(\xi, \xi)_{x}=\alpha\right\}$. Assume that the sectional curvature $K_{g} \leqq 1$ and $\beta=\max _{x \in M} d_{g}(x, \mathscr{F})<\pi / 2$. Then for any point $p$ of $M$ we obtain

(i) $g(\xi, \xi)_{p} \geqq \alpha \cos ^{2} \beta$,

(ii) $\left\|(\operatorname{grad} g(\xi, \xi))_{p}\right\| \leqq 2 \alpha \sin \beta$, where \|\| denotes $g(,)^{1 / 2}$.

In order to prove the theorem, we provide the following propositions.

Proposition 2.2. Let $f$ be a positive differentiable function defined in the interval $\left(s_{1}, s_{2}\right)$ such that $-\pi / 2<s_{1}<0<s_{2}<\pi / 2, \max f=f(0)$ and $f^{\prime \prime}(s) \geqq-f(s)$. Then $f(s) \geqq f(0) \cos s$.

Proof. Let $\varepsilon$ be a positive number. Put $f_{\varepsilon}(s)=(f(0)+\varepsilon) \cos s /(f(s)+\varepsilon)$. Then we obtain

$$
\begin{aligned}
& f_{\varepsilon}^{\prime}(s)=\frac{-(f(0)+\varepsilon)(f(s)+\varepsilon) \sin s-(f(0)+\varepsilon) f^{\prime}(s) \cos s}{(f(s)+\varepsilon)^{2}} \\
& f_{\varepsilon}^{\prime \prime}(s)=-\frac{(f(0)+\varepsilon)\left(f(s)+\varepsilon+f^{\prime \prime}(s)\right) \cos s}{(f(s)+\varepsilon)^{2}}-\frac{2 f_{\varepsilon}^{\prime}(s) f^{\prime}(s)}{f(s)+\varepsilon} .
\end{aligned}
$$


Since $f_{\varepsilon}^{\prime}(0)=0$ and $f_{\varepsilon}^{\prime \prime}(0)<0, f_{\varepsilon}$ is maximal at 0 . If $f_{\varepsilon}^{\prime}\left(s_{0}\right)=0$ for some $s_{0}$, then it follows that

$$
f_{\varepsilon}^{\prime \prime}\left(s_{0}\right)=-\frac{(f(0)+\varepsilon)\left(f\left(s_{0}\right)+\varepsilon+f^{\prime \prime}\left(s_{0}\right)\right) \cos s_{0}}{\left(f\left(s_{0}\right)+\varepsilon\right)^{2}}<0 .
$$

Hence every critical point of $f_{\varepsilon}$ in the interval $\left(s_{1}, s_{2}\right)$ is maximal, which implies that $f_{\varepsilon}$ has no critical points in $\left(s_{1}, s_{2}\right)$ except at 0 . Therefore we obtain $f_{\varepsilon}(s)$ $\leqq f_{\varepsilon}(0)=1$, i.e., $(f(0)+\varepsilon) \cos s \leqq f(s)+\varepsilon$. By $\varepsilon$ passing to 0 , the assertion is implied.

Proposition 2.3. Let $f: \mathbb{R} \rightarrow \mathbb{R}$ be a positive differentiable function such that $f^{\prime \prime}(s) \geqq-f(s) \geqq-\alpha$, where $\alpha$ is a positive number. Then $f^{\prime}(s)$ $\leqq \sqrt{2 \alpha(\alpha-f(s))}$.

Proof. Since $f^{\prime \prime}(s) \geqq-\alpha$, we have for any $t>s$

$$
\begin{aligned}
-\frac{1}{2}(t-s)^{2} \alpha & \leqq \int_{s}^{t}\left(\int_{s}^{\tau} f^{\prime \prime}(\sigma) d \sigma\right) d \tau \\
& =f(t)-f(s)-(t-s) f^{\prime}(s) .
\end{aligned}
$$

It implies

$$
f^{\prime}(s) \leqq \frac{1}{2}(t-s) \alpha+\frac{\alpha-f(s)}{t-s} .
$$

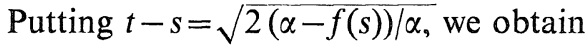

$$
f^{\prime}(s) \leqq \sqrt{2 \alpha(\alpha-f(s))} .
$$

Proof of Theorem 2.1. Let $\gamma: \mathbb{R} \rightarrow M$ be a geodesic with $\|\dot{\gamma}\|=1$. Since $\xi$ is a Killing vector field, it satisfies

$$
\frac{1}{2} \dot{\gamma} \dot{\gamma} g(\xi, \xi)_{\gamma(t)}=g\left(\nabla_{\dot{\gamma}} \xi, \nabla_{\dot{\gamma}} \xi\right)_{\gamma(t)}-g(R(\dot{\gamma}, \xi) \xi, \dot{\gamma})_{\gamma(t)},
$$

where $\dot{\gamma}$ denotes the velocity vector of $\gamma$ and $R$ is the curvature tensor of the Riemannian connection of $g$.

(i) Put $f(s)=\left\|\xi_{\gamma(s)}\right\|$ and $F=\{s ; f(s)=0\}$. We define $E_{\gamma(s)}=\xi_{\gamma(s)} / f(s)$ for $s \notin F$. Then from (2.1) we obtain

$$
f(s) f^{\prime \prime}(s)=(f(s))^{2} g\left(\nabla_{\dot{\gamma}} E, \nabla_{\dot{\gamma}} E\right)-(f(s))^{2} g(R(\dot{\gamma}, E) E, \dot{\gamma}) .
$$

Since the sectional curvature $K_{g} \leqq 1$, we obtain

$$
f^{\prime \prime}(s) \geqq-f(s) \quad \text { for } \quad s \notin F .
$$


There is a point $q$ in $\mathscr{F}$ such that $d_{g}(p, q)=d_{g}(p, \mathscr{F})$. Let $\gamma:\left[0, s_{0}\right] \rightarrow M$ be a minimal geodesic from $q$ to $p$, i.e., $d_{g}(q, p)=s_{0}<\pi / 2$. First we show that $f(s) \neq 0\left(s \in\left[0, s_{0}\right]\right)$. Suppose that $F \cap\left[0, s_{0}\right] \neq \varnothing$. Put inf $F \cap\left[0, s_{0}\right]=s_{1}$. Then $s_{1}>0$ and $s_{1} \in F$. From (2.2) and Proposition 2.2, we obtain $f(s)$ $\geqq f(0) \cos s\left(s \in\left[0, s_{1}\right]\right)$. Hence it follows that

$$
f\left(s_{1}\right) \geqq f(0) \cos s_{1} \geqq f(0) \cos s_{0}>0,
$$

which contradicts $s_{1} \in F$. Therefore we obtain $F \cap\left[0, s_{0}\right]=\varnothing$. Hence (i) follows from Proposition 2.2.

(ii) Let $\gamma(0)=p$. Put $f(s)=\left\|\xi_{\gamma(s / \sqrt{2})}\right\|^{2}$. Then from (i) we obtain

$$
f(s) \geqq \alpha \cos ^{2} \beta>0 \text {. }
$$

On the other hand, from (2.1) and $K_{g} \leqq 1$, we obtain

$$
f^{\prime \prime}(s) \geqq-f(s) \text {. }
$$

Hence it follows from Proposition 2.3 that

$$
\begin{aligned}
\dot{\gamma} g(\xi, \xi)_{\gamma(0)} & =\sqrt{2} f^{\prime}(s) \\
& \leqq 2 \sqrt{\alpha(\alpha-f(s))} \\
& \leqq 2 \alpha \sin \beta .
\end{aligned}
$$

We note that

$$
g\left(\dot{\gamma},(\operatorname{grad} g(\xi, \xi))_{p}\right)=\dot{\gamma} g(\xi, \xi)_{p} .
$$

Since we can choose the direction of $\gamma$ at $\gamma(0)=p$ arbitrarily, our assertion is clear.

Q.E.D.

\section{§3. The Sectional Curvature of Invariant Metrics on a Lie Group}

Let $G$ be a compact connected Lie group with a left-invariant Riemannian metric $g$. We denote by $g$ the tangent space to $G$ at the identity $e$. Let $X$ be a tangent vector to $G$ at $e$. We denote by $X^{L}$ the left-invariant vector field on $G$ such that the value $X_{e}^{L}$ of $X^{L}$ at $e$ is $X$. We also define a right-invariant vector field $X^{R}$ similarly. We denote by $\mathfrak{g}^{L}$ the Lie algebra of left-invariant vector fields on $G$.

A bi-linear form $U(g): \mathfrak{g}^{L} \times \mathfrak{g}^{L} \rightarrow \mathfrak{g}^{L}$ is defined by

$$
2 g\left(U(g)\left(X^{L}, Y^{L}\right), Z^{L}\right)=g\left(X^{L},\left[Z^{L}, Y^{L}\right]\right)+g\left(Y^{L},\left[Z^{L}, X^{L}\right]\right)
$$

$(X, Y, Z \in \mathfrak{g})$. We note that the Riemannian connection $\nabla$ of $g$ has an 
expression

$$
\nabla_{X^{L}} Y^{L}=U(g)\left(X^{L}, Y^{L}\right)+\frac{1}{2}\left[X^{L}, Y^{L}\right] \quad(X, Y \in \mathfrak{g})
$$

and the curvature tensor $R(g)$ of $\nabla$ satisfies

$$
\begin{aligned}
g(R(g)(X, Y) Y, X)= & \left\|U(g)\left(X^{L}, Y^{L}\right)_{e}\right\|^{2}-g\left(U(g)\left(X^{L}, X^{L}\right)_{e}, U(g)\left(Y^{L}, Y^{L}\right)_{e}\right) \\
& -\frac{3}{4}\left\|\left[X^{L}, Y^{L}\right]_{e}\right\|^{2}-\frac{1}{2} g\left(\left[X^{L},\left[X^{L}, Y^{L}\right]\right]_{e}, Y_{e}^{L}\right) \\
& -\frac{1}{2} g\left(\left[Y^{L},\left[Y^{L}, X^{L}\right]\right]_{e}, X_{e}^{L}\right) .
\end{aligned}
$$

Lemma 3.1. $U(g)\left(X^{L}, Y^{L}\right)_{e}=-\frac{1}{2}\left(\operatorname{grad} g\left(X^{R}, Y^{R}\right)\right)_{e} \quad(X, Y \in \mathfrak{g})$.

Proof. For a vector $Z \in \mathfrak{g}$, we obtain

$$
\begin{aligned}
g\left(U(g)\left(X^{L}, Y^{L}\right)_{e}, Z\right) & =\frac{1}{2}\left\{g\left(\nabla_{X^{L}} Y^{L}, Z^{L}\right)_{e}+g\left(\nabla_{Y^{L}} X^{L}, Z^{L}\right)_{e}\right\} \\
& =-\frac{1}{2}\left\{g\left(Y^{L}, \nabla_{X^{L}} Z^{L}\right)_{e}+g\left(X^{L}, \nabla_{Y^{L}} Z^{L}\right)_{e}\right\} \\
& =-\frac{1}{2}\left\{g\left(Y^{R}, \nabla_{X^{R}} Z^{L}\right)_{e}+g\left(X^{R}, \nabla_{Y^{R}} Z^{L}\right)_{e}\right\} \\
& =-\frac{1}{2}\left\{g\left(Y^{R}, \nabla_{Z^{L}} X^{R}\right)_{e}+g\left(X^{R}, \nabla_{Z^{L}} Y^{R}\right)_{e}\right\} \\
& =-\frac{1}{2} Z g\left(Y^{R}, X^{R}\right) .
\end{aligned}
$$

Let $a$ be an element of $G$. We denote by $R_{a}$ the right translation by $a$. Let $d v$ be a bi-invariant volume element on $G$ with $\int_{G} d v=1$. We define a biinvariant Riemannian metric $\tilde{g}$ on $G$ by

$$
\tilde{g}=\int_{a \in G} R_{a}^{*} g d v .
$$

Let $H$ be a finite subgroup of $G$ such that $g$ is invariant by the right action of $H$. Then there is a Riemannian metric on $G / H$ such that the projection $(G, g)$ $\rightarrow G / H$ is a Riemannian covering. We denote the metric also by $g$. We also define a Riemannian manifold $(G / H, \tilde{g})$ in like manner. The diameter of $(G / H, g)$ (resp. $(G / H, \tilde{g}))$ is denoted by $d_{g}(G / H)$ (resp. $\left.d_{\tilde{g}}(G / H)\right) . \quad K_{g}$ denotes the sectional curvature of $(G, g)$.

Lemma 3.2. Assume that $K_{g} \leqq 1$ and $d_{g}(G / H)<\pi / 2$. Then for any $X$ $(\in \mathfrak{g}, \neq 0)$

$$
\cos ^{2} d_{g}(G / H) \leqq \frac{\tilde{g}(X, X)}{g(X, X)} \leqq\left(\cos d_{g}(G / H)\right)^{-2}
$$


Proof. By definition we obtain

$$
\tilde{g}(X, X)=\int_{a \in G} g\left(X^{R}, X^{R}\right)_{a} d v .
$$

Since $X^{R}$ is a Killing vector field on $(G, g)$ and $g\left(X^{R}, X^{R}\right)$ is constant on each right orbit $a H$ of $H$, it follows from Theorem 2.1 that

$$
\begin{aligned}
g(X, X) \cos ^{2} d_{g}(G / H) & =g\left(X^{R}, X^{R}\right)_{e} \cos ^{2} d_{g}(G / H) \\
& \leqq \max _{x \in G} g\left(X^{R}, X^{R}\right)_{x} \cos ^{2} d_{g}(G / H) \\
& \leqq g\left(X^{R}, X^{R}\right)_{a} \quad(\forall a \in G) \\
& \leqq \max _{x \in G} g\left(X^{R}, X^{R}\right)_{x} \\
& \leqq g\left(X^{R}, X^{R}\right)_{e}\left(\cos d_{g}(G / H)\right)^{-2} \\
& =g(X, X)\left(\cos d_{g}(G / H)\right)^{-2}
\end{aligned}
$$

Hence the assertion is clear.

Q.E.D.

Since both metrics $g$ and $\tilde{g}$ on $G$ are left-invariant, from Lemma 3.2 we obtain

Lemma 3.3. If $K_{g} \leqq 1$ and $d_{g}(G / H)<\pi / 2$, then

$$
\cos d_{g}(G / H) \leqq \frac{d_{\tilde{g}}(G / H)}{d_{g}(G / H)} \leqq\left(\cos d_{g}(G / H)\right)^{-1} .
$$

Lemma 3.4. Let $a$ be an element of $G$. Let $X, Y(\in \mathfrak{g})$ be linearly independent vectors such that $\tilde{g}(X, X)=\tilde{g}(Y, Y)=1$. Assume that $K_{g} \leqq 1$ and $d_{g}(G / H)<\pi / 2$. Then

$$
\begin{aligned}
& \left(R_{a}^{*} g\right)\left(U\left(R_{a}^{*} g\right)\left(X^{L}, X^{L}\right)_{e}, U\left(R_{a}^{*} g\right)\left(X^{L}, X^{L}\right)_{e}\right)^{1 / 2} \\
& \quad \leqq\left(\cos d_{g}(G / H)\right)^{-2} \sin d_{g}(G / H), \\
& \left(R_{a}^{*} g\right)\left(R\left(R_{a}^{*} g\right)(X, Y) Y, X\right) \leqq\left(\cos d_{g}(G / H)\right)^{-4} .
\end{aligned}
$$

Proof. Since we have

$$
\tilde{\mathfrak{g}}(X, X)=\int_{x \in G} g\left(X^{R}, X^{R}\right)_{x} d v=1,
$$

there is a point $p$ in $G$ such that $g\left(X^{R}, X^{R}\right)_{p}=1$. Since $X^{R}$ is a Killing vector field on $(G, g)$ such that $g\left(X^{R}, X^{R}\right)$ is constant on each right orbit $x H$ of $H$ $(x \in G)$, it follows from Theorem 2.1 that

$$
\max _{x \in G} g\left(X^{R}, X^{R}\right)_{x} \leqq g\left(X^{R}, X^{R}\right)_{p}\left(\cos d_{g}(G / H)\right)^{-2}=\left(\cos d_{g}(G / H)\right)^{-2} .
$$

Similarly we obtain 


$$
\max _{x \in G} g\left(Y^{R}, Y^{R}\right)_{x} \leqq\left(\cos d_{g}(G / H)\right)^{-2} .
$$

(i) Since $(G / H, g)$ and $\left(G / a H a^{-1}, R_{a}^{*} g\right)$ are isometric, $K_{R_{a}^{*} g} \leqq 1$ and $d_{R_{a}^{*} g}\left(G / a H a^{-1}\right)=d_{g}(G / H)<\pi / 2$. Since $X^{R}$ is a Killing vector field also on $\left(G, R_{a}^{*} g\right)$ such that $\left(R_{a}^{*} g\right)\left(X^{R}, X^{R}\right)$ is constant on each right orbit of $a H a^{-1}$, it follows from Lemma 3.1 and Theorem 2.1 that

$$
\begin{aligned}
& \left(R_{a}^{*} g\right)\left(U\left(R_{a}^{*} g\right)\left(X^{L}, X^{L}\right)_{e}, U\left(R_{a}^{*} g\right)\left(X^{L}, X^{L}\right)_{e}\right)^{1 / 2} \\
& \quad=\frac{1}{2}\left(R_{a}^{*} g\right)\left(\left(\operatorname{grad}_{R_{a}^{*} g}\left(R_{a}^{*} g\right)\left(X^{R}, X^{R}\right)\right)_{e},\left(\operatorname{grad}_{R_{a}^{*} g}\left(R_{a}^{*} g\right)\left(X^{R}, X^{R}\right)\right)_{e}\right)^{1 / 2} \\
& \quad \leqq \max _{x \in G}\left(R_{a}^{*} g\right)\left(X^{R}, X^{R}\right)_{x} \sin d_{g}(G / H) \\
& \quad=\max _{x \in G} g\left(X^{R}, X^{R}\right)_{x} \sin d_{g}(G / H) \\
& \quad \leqq\left(\cos d_{g}(G / H)\right)^{-2} \sin d_{g}(G / H) .
\end{aligned}
$$

(ii) Since $g$ and $R_{a}^{*} g$ are isometric, we obtain

$$
1 \geqq K_{R_{a}^{*} g}(X, Y)=\frac{\left(R_{a}^{*} g\right)\left(R\left(R_{a}^{*} g\right)(X, Y) Y, X\right)}{\left(R_{a}^{*} g\right)(X, X)\left(R_{a}^{*} g\right)(Y, Y)-\left(R_{a}^{*} g\right)(X, Y)^{2}} .
$$

Hence

$$
\begin{array}{rlr}
\left(R_{a}^{*} g\right)\left(R\left(R_{a}^{*} g\right)(X, Y) Y, X\right) & \leqq\left(R_{a}^{*} g\right)(X, X)\left(R_{a}^{*} g\right)(Y, Y) \\
& =g\left(X^{R}, X^{R}\right)_{a} g\left(Y^{R}, Y^{R}\right)_{a} & \\
& \leqq\left(\cos d_{g}(G / H)\right)^{-4} . & \text { Q. E. D. }
\end{array}
$$

Theorem 3.5. Assume that the sectional curvature $K_{g} \leqq 1$ and the diameter $d_{g}(G / H)<\pi / 2$. Then the sectional curvature $K_{\tilde{g}}$ of $\tilde{g}$ satisfies

$$
K_{\tilde{g}} \leqq\left(\cos d_{g}(G / H)\right)^{-4}\left(1+\sin ^{2} d_{g}(G / H)\right) .
$$

Proof. Since $\tilde{g}$ is bi-invariant, $U(\tilde{g}) \equiv 0$ (cf. Lemma 3.1). We take vectors $X, Y(\in \mathfrak{g})$ such that $\tilde{g}(X, X)=\tilde{g}(Y, Y)=1$ and $\tilde{g}(X, Y)=0$. Then from Lemma 3.4 we obtain

$$
\begin{aligned}
K_{\tilde{g}}(X, Y)= & \frac{\tilde{g}(R(\tilde{g})(X, Y) Y, X)}{\tilde{g}(X, X) \tilde{g}(Y, Y)-\tilde{g}(X, Y)^{2}} \\
= & -\frac{3}{4} \tilde{g}\left(\left[X^{L}, Y^{L}\right]_{e},\left[X^{L}, Y^{L}\right]_{e}\right) \\
& -\frac{1}{2} \tilde{g}\left(\left[X^{L},\left[X^{L}, Y^{L}\right]\right]_{e}, Y_{e}^{L}\right)-\frac{1}{2} \tilde{g}\left(\left[Y^{L},\left[Y^{L}, X^{L}\right]\right]_{e}, X_{e}^{L}\right) \\
= & \int_{a \in G}\left\{-\frac{3}{4}\left(R_{a}^{*} g\right)\left(\left[X^{L}, Y^{L}\right]_{e},\left[X^{L}, Y^{L}\right]_{e}\right)\right. \\
& -\frac{1}{2}\left(R_{a}^{*} g\right)\left(\left[X^{L},\left[X^{L}, Y^{L}\right]_{e}, Y_{e}^{L}\right)\right.
\end{aligned}
$$




$$
\begin{aligned}
& \left.-\frac{1}{2}\left(R_{a}^{*} g\right)\left(\left[Y^{L},\left[Y^{L}, X^{L}\right]\right]_{e}, X_{e}^{L}\right)\right\} d v \\
= & \int_{a \in G}\left\{\left(R_{a}^{*} g\right)\left(R\left(R_{a}^{*} g\right)(X, Y) Y, X\right)\right. \\
& -\left(R_{a}^{*} g\right)\left(U\left(R_{a}^{*} g\right)\left(X^{L}, Y^{L}\right)_{e}, U\left(R_{a}^{*} g\right)\left(X^{L}, Y^{L}\right)_{e}\right) \\
& \left.+\left(R_{a}^{*} g\right)\left(U\left(R_{a}^{*} g\right)\left(X^{L}, X^{L}\right)_{e}, U\left(R_{a}^{*} g\right)\left(Y^{L}, Y^{L}\right)_{e}\right)\right\} d v \\
\leqq & \left(\cos d_{g}(G / H)\right)^{-4}+\sin ^{2} d_{g}(G / H)\left(\cos d_{g}(G / H)\right)^{-4} .
\end{aligned}
$$

\section{§4. Bi-invariant Metrics and Finite Subgroups of a Lie Group}

Let $G$ be a compact connected Lie group as in Section 3. Let exp denote the usual exponential mapping from $\mathfrak{g}$ to $G$, i.e., for a tangent vector $X \in \mathfrak{g} \gamma(t)=$ $\exp t X(t \in \boldsymbol{R})$ is a one-parameter subgroup of $G$ such that $\dot{\gamma}(0)=X$. The usual bracket operation is defined by

$$
[X, Y]=\left.\frac{d}{d t}(\operatorname{Ad}(\exp t X) Y)\right|_{t=0}
$$

$X, Y \in \mathfrak{g}$. Let $\tilde{g}$ be a bi-invariant Riemannian metric on $G$ with sectional curvature $K_{\tilde{g}} \leqq k(k>0)$. We note the mapping exp: $\mathfrak{g} \rightarrow G$ coincides with the usual exponential mapping of the Riemannian manifold $(G, \tilde{g})$ because the metric $\tilde{g}$ is bi-invariant. For non-zero vectors $X$ and $Y$ of $\mathfrak{g}$ we denote by $\Varangle(X, Y)$ the angle which $X$ and $Y$ make. \|\| denotes $\tilde{g}(,)^{1 / 2}$.

Lemma 4.1. Let $X$ and $Y$ be non-zero vectors of $\mathrm{g}$. We have

$$
\Varangle(\operatorname{Ad}(\exp Y) X, X) \leqq \frac{\|[Y, X]\|}{\|X\|} .
$$

Proof. Since the metric $\tilde{g}$ is bi-invariant and $\left.\frac{d}{d t} \operatorname{Ad}(\exp t Y) X\right|_{t=0}$ $=[Y, X]$, we see $\|\operatorname{Ad}(\exp t Y) X\|=\|X\|$ and $\left\|\frac{d}{d t} \operatorname{Ad}(\exp t Y) X\right\|=\|[Y, X]\|$. Hence it follows that

$$
\begin{aligned}
\Varangle(\operatorname{Ad}(\exp Y) X, X) & \leqq \frac{1}{\|X\|} \int_{0}^{1}\left\|\frac{d}{d t} \operatorname{Ad}(\exp t Y) X\right\| d t \\
& =\frac{\|[Y, X]\|}{\|X\|} .
\end{aligned}
$$

Lemma 4.2. Let $X$ and $Y$ be non-zero vectors of $\mathfrak{g}$ such that $\exp t X$ $(0 \leqq t \leqq 1)$ and $\exp t Y(0 \leqq t \leqq 1)$ are minimal geodesics. Suppose that $\|X\|$ $=d_{\tilde{g}}(e, \exp X)<\pi / \sqrt{k}$ and $\|[X, Y]\| /\|X\|<\pi / 3$. Then 


$$
d_{\tilde{g}}(e, \exp X)>d_{\tilde{g}}\left(\exp X, \exp Y \exp X \exp Y^{-1}\right) .
$$

Remark. The similar estimate is found in [1].

Proof. From Lemma 4.1, we obtain $\Varangle(\operatorname{Ad}(\exp Y) X, X)<\pi / 3$. It implies $\|X\|>\|X-\operatorname{Ad}(\exp Y) X\|$. Let us define a curve $\gamma:[0,1] \rightarrow \mathfrak{g}$ by $\gamma(t)=t X$ $+(1-t) \operatorname{Ad}(\exp Y) X$. We have the sectional curvature $K_{\tilde{g}} \geqq 0$ since the metric $\tilde{g}$ is bi-invariant. From Rauch's comparison theorem we easily see that

$$
\begin{aligned}
d_{\tilde{g}}(e, \exp X) & =\|X\| \\
& \geqq\|X-\operatorname{Ad}(\exp Y) X\| \\
& =\text { length }(\gamma) \\
& \geqq \text { length }(\exp \circ \gamma) \\
& \geqq d_{\tilde{g}}\left(\exp X, \exp Y \exp X \exp Y^{-1}\right) .
\end{aligned}
$$

Lemma 4.3. Let $c(\neq e)$ be an element of the center of $G$. If $G$ is semisimple, then $d_{\tilde{g}}(e, c) \geqq \pi / \sqrt{k}$.

Proof. Let $\gamma:[0,1] \rightarrow G$ be a mininal geodesic from $e$ to $c$. Then $\gamma$ is expressed as $\gamma(t)=\exp t Y$ for somc $X \in \mathfrak{g}$. Since $G$ is semi-simple, the orbit $\operatorname{Ad}(G) X(\subset g)$ of $X$ by the adjoint action of $G$ is at least of one dimension. Since $\exp \operatorname{Ad}(G) X=c, c$ is conjugate to $e$ along $\gamma$. Hence the assertion follows from the Morse-Shoenberg theorem.

Q.E.D.

Let $x$ be a point of $G . \quad C(x)$ denotes the cut locus of $x$ with respect to the metric $\tilde{g}$.

Lemma 4.4. If $G$ is semi-simple, then $d_{\tilde{g}}(e, C(e)) \geqq \pi / 2 \sqrt{k}$.

Proof. Since the metric $\tilde{g}$ is bi-invariant, the isotropy $\operatorname{subgroup} I_{e}(G, \tilde{g})$ at $e$ contains the inner automorphisms $\operatorname{Ad}(G)$ of $G$. Since the fixed points $F(\operatorname{Ad}(G))$ is the center of $G$ and since the center consists of finite points, the assertion follows from Lemma 1.1.

Q.E.D.

Let $H$ be a finite subgroup of $G$. Let $h$ be an element of $H \backslash\{e\}$ which is the closest to e. Put $Z(h)=\{x \in G ; x h=h x\}$.

Lemma 4.5. If $G$ is semi-simple and $d_{\tilde{g}}(e, h)<\pi / 2, / \bar{k}$, then $Z(h) \varsubsetneqq G$ and $\max _{x \in G} d_{\tilde{q}}(x, Z(h)) \geqq \pi / 2 \sqrt{k}$.

Proof. From Lemma 4.4, we see that the minimal geodesic from $e$ to $h$ is unique. We denote the geodesic by $\exp t X(0 \leqq t \leqq 1)$, where $X \in \mathfrak{g}$. Since for any $x \in Z(h)$ the inner automorphism by $x$ fixes the endpoints of the geodesic and 
since the minimal geodesic connecting $e$ and $h$ is unique, we obtain $x(\exp t X) x^{-1}$ $=\exp t X(0 \leqq t \leqq 1)$, which implies that

$$
\begin{aligned}
Z(h) & =\left\{x \in G ;(\exp t X) x(\exp t X)^{-1}=x \quad(t \in \boldsymbol{R})\right\} \\
& =F\left(\left\{L_{\exp t X}\left(R_{\exp t X}\right)^{-1} ; t \in \boldsymbol{R}\right\}\right) .
\end{aligned}
$$

Since $G$ is semi-simple, the assertion follows from Lemma 1.1.

Q.E.D.

Lemma 4.6. Suppose that $H \nsubseteq Z(h)$. Then for any $a \in H \backslash Z(h) d_{\hat{g}}(e, a)$ $\geqq \pi / 6 \sqrt{k}$.

Proof. Let $\gamma, \delta:[0,1] \rightarrow G$ be minimal geodesics from $e$ to $h$ and $a$ respectively. Then there are $X$ and $Y$ in $g$ such that $\gamma(t)=\exp t X$ and $\delta(t)=\exp t Y$. If $\|[X, Y]\| /\|X\|<\pi / 3$, Lemma 4.2 implies that

$$
d_{\tilde{g}}(e, h)>d_{\tilde{g}}\left(h, a h a^{-1}\right)=d_{\tilde{g}}\left(e, a h a^{-1} h^{-1}\right),
$$

which contradicts the choice of $h$. Hence we obtain $\|[X, Y]\| /\|X\| \geqq \pi / 3$ On the other hand, we have

$$
k \geqq K_{\tilde{g}}(X, Y)=\frac{\frac{1}{4}\|[X, Y]\|^{2}}{\|X\|^{2}\|Y\|^{2}-\tilde{g}(X, Y)^{2}} .
$$

Hence it follows that

$$
k \geqq \frac{\frac{1}{4}\|[X, Y]\|^{2}}{\|X\|^{2}\|Y\|^{2}} \geqq \frac{\pi^{2}}{36\|Y\|^{2}},
$$

which implies

$$
d_{\tilde{g}}(e, a)=\|Y\| \geqq \frac{\pi}{6 \sqrt{k}}
$$

Theorem 4.7. Assume that the group $G$ is not abelian. Then the following (i), (ii) and (iii) hold.

(i) $\quad d_{\tilde{g}}(G) \geqq \frac{\pi}{2 \sqrt{k}}$.

(ii) If $G$ is simply connected, then $d_{\tilde{g}}(G) \geqq \frac{\pi}{\sqrt{k}}$.

(iii) $\max _{x \in G} d_{\tilde{g}}(x, H)=d_{\tilde{g}}(G / H) \geqq \frac{\pi}{12 \sqrt{k}}$.

Proof. Let $Z$ be the identity component of the center of $G$. We put $G^{\prime}$ $=G / Z$. Then $G^{\prime}$ is semi-simple and if $G$ is simply connected, so is $G^{\prime}$. The metric $\tilde{g}$ on $G$ induces a Riemannian metric $\tilde{g}^{\prime}$ on $G^{\prime}$ so that the projection 
$\pi: G \rightarrow G^{\prime}$ is a Riemannian submersion. Let $z$ denote the tangent space to $Z$ at $e$. We take orthonormal vectors $X$ and $Y$ in $g$ such that $X, Y \perp z$. Then from O'Neil's theorem for Riemannian submersion we obtain

$$
K_{\tilde{g}^{\prime}}\left(\pi_{*} X, \pi_{*} Y\right)=K_{\tilde{g}}(X, Y)+\frac{3}{4}\left\|[X, Y]_{\tilde{\jmath}}\right\|^{2},
$$

where $[X, Y]_{3}$ denotes the orthogonal projection of $[X, Y]$ to 3 . Since $[X, Y]$ $\perp_{3}$, we obtain $K_{\tilde{g}^{\prime}} \leqq k$. On the other hand it is clear that, for any point $x$ and $y$ of $G, d_{\tilde{g}}(x, y) \geqq d_{\tilde{g}^{\prime}}(\pi(x), \pi(y))$. Hence we have only to prove the theorem with the assumption that $G$ is semi-simple. So we suppose $G$ is semi-simple.

(i) From Lemma 4.4, we obtain

$$
d_{\tilde{g}}(G) \geqq d_{\tilde{g}}(e, C(e)) \geqq \frac{\pi}{2 \sqrt{k}} .
$$

(ii) Corollary 5.12 in [2] states that the cut locus and first conjugate locus coincide. The assertion follows easily from the Morse-Shoenberg theorem.

(iii) If $H=\{e\}$, the inequality follows from (i). Hence we assume that $H \supsetneqq$ $\{e\}$. Let $h$ be an element of $H \backslash\{e\}$ which is the closest to $e$. Let $m$ be the middle point of a minimal geodesic from $e$ to $h$. Then it is easy to see that $d_{\hat{g}}(m, H)$ $=d_{\tilde{g}}(m, e)=\frac{1}{2} d_{\tilde{g}}(e, h)$. If $d_{\tilde{g}}(e, h) \geqq \pi / 2 \sqrt{k}$, then $\max _{x \in G} d_{\tilde{g}}(x, H) \geqq d_{\tilde{g}}(m, H)$ $\geqq \pi / 4 \sqrt{k}$. Hence we may assume that $d_{\tilde{g}}(e, h)<\pi / 2 \sqrt{k}$. If $H \subset Z(h)$, the inequality follows from Lemma 4.5. Therefore we suppose that $H \nsubseteq Z(h)$. Let $a$ be an element of $H \backslash Z(h)$ which is the closest to $e$. Let $m^{\prime}$ be the middle point of a minimal geodesic from $e$ to $a$. Then we have $d_{\tilde{g}}\left(m^{\prime}, H\right)=d_{\tilde{g}}\left(m^{\prime}, e\right)$. (In fact, suppose that there is an element $b \in H$ with $d_{\tilde{g}}\left(m^{\prime}, b\right)<d_{\tilde{g}}\left(m^{\prime}, e\right)$. Then it follows from $d_{\tilde{g}}(e, b) \leqq d_{\tilde{g}}\left(e, m^{\prime}\right)+d_{\tilde{g}}\left(m^{\prime}, b\right)<d_{\tilde{g}}(e, a)$ that $b \in Z(h)$. Hence we obtain $a b^{-1} \in H \backslash Z(h)$ and $d_{\tilde{g}}\left(e, a b^{-1}\right)=d_{\tilde{g}}(b, a) \leqq d_{\tilde{g}}\left(b, m^{\prime}\right)+d_{\tilde{g}}\left(m^{\prime}, a\right)<d_{\tilde{g}}(e, a)$, which means that $a$ is not the closest to $e$ of $H \backslash Z(h)$.) Therefore the inequality follows from Lemma 4.6.

Q.E.D.

\section{§5. Diameter Estimate}

Theorem 5.1. Let $(M, g)$ be a compact homogeneous Riemannian manifold with the sectional curvature $K_{g} \leqq 1$ and $K_{g} \not \equiv 0$. Then the diameter $d_{g}(M)$ of $(M, g)$ is not less than a positive constant $d(>0.23)$.

Proof. Let $p$ be a point of $M$. If $\operatorname{dim} I_{p}(M, g) \geqq 1$, then from Lemma 1.1 we obtain 


$$
d_{g}(M) \geqq \max _{x \in M} d_{g}\left(x, F\left(I_{p}^{\circ}(M, g)\right)\right) \geqq \frac{\pi}{2},
$$

where $I_{p}^{\circ}(M, g)$ denotes the identity component of $I_{p}(M, g)$ (see also [3]).

Hence we assume that $\operatorname{dim} I_{p}(M, g)=0$. We denote by $G$ the identity component of $I(M, g)$ and put $H=G \cap I_{p}(M, g)$. Since the projection $G \rightarrow G / H=M$ is a covering, $g$ induces a left-invariant Riemannian metric on $G$ such that the projection is a Riemannian covering. We denote the metric also by $g$. It is invariant by the inner automorphism by $H$. We define a Riemannian metric $\tilde{g}$ as in Section 3. Suppose that $d_{g}(G / H)<\pi / 2$. Then from Theorem 3.5 we obtain

$$
K_{\tilde{g}} \leqq\left(\cos d_{g}(G / H)\right)^{-4}\left(1+\sin ^{2} d_{g}(G / H)\right) .
$$

Since $K_{g} \not \equiv 0$, it is easily seen that $G$ is not abelian. Hence it follows from Lemma 3.3 and Theorem 4.7 that

$$
\begin{aligned}
\frac{\pi}{12 \sqrt{\left(\cos d_{g}(G / H)\right)^{-4}\left(1+\sin ^{2} d_{g}(G / H)\right)}} & \leqq d_{\tilde{g}}(G / H) \\
& \leqq d_{g}(G / H)\left(\cos d_{g}(G / H)\right)^{-1}
\end{aligned}
$$

We put

$$
d=\inf \left\{t \geqq 0 ; \frac{\pi}{12} \leqq t(\cos t)^{-3}\left(1+\sin ^{2} t\right)^{1 / 2}\right\} .
$$

Then $\pi / 2>d>0.23$ and $d_{g}(M) \geqq d$.

Q.E.D.

Theorem 5.2. Let $(M, g)$ be a simply connected compact homogeneous Riemannian manifold with sectional curvature $K_{g} \leqq 1$ and $K_{g} \neq 0$. Then the diameter $d_{g}(M)$ of $(M, g)$ is not less than a positive constant $d_{0}(>0.81)$.

Proof. As in the proof of Theorem 5.1, we may assume that $\operatorname{dim} I_{p}(M, g)$ $=0$. We define $G$ and $H$ as in the proof of Theorem 5.1. Since $M$ is simply connected, $H=\{e\}$ and $G=M$. Hence it follows from Lemma 3.3 and (i) of Theorem 4.7 that we can replace $(5.1)$ by

$$
\frac{\pi}{\sqrt{\left(\cos d_{g}(G)\right)^{-4}\left(1+\sin ^{2} d_{g}(G)\right)}} \leqq d_{g}(G)\left(\cos d_{g}(G)\right)^{-1}
$$

We put

$$
d_{0}=\inf \left\{t \geqq 0 ; \pi \leqq t(\cos t)^{-3}\left(1+\sin ^{2} t\right)^{1 / 2}\right\} .
$$

Then $\pi / 2>d_{0}>0.81$ and $d_{g}(M)=d_{g}(G) \geqq d_{0}$.

Theorem 5.3. Let $G$ be a compact connected Lie group with a left- 
invariant metric $g$. Assume that the sectional curvature $K_{g} \leqq 1$ and $G$ is not abelian. Then the diameter $d_{g}(G)$ of $(G, g)$ is not less than a positive constant $d_{1}(>0.66)$.

Proof. We define a metric $\tilde{g}$ as in Section 3. We may assume that $d_{g}(G)<\pi / 2$. From Theorem 3.5, we obtain

$$
K_{\tilde{g}} \leqq\left(\cos d_{g}(G)\right)^{-4}\left(1+\sin ^{2} d_{g}(G)\right) .
$$

Hence it follows from Lemma 3.3 and Theorem 4.7 that

$$
\begin{aligned}
\frac{\pi}{2 \sqrt{\left(\cos d_{g}(G)\right)^{-4}\left(1+\sin ^{2} d_{g}(G)\right)}} & \leqq d_{\tilde{g}}(G) \\
& \leqq d_{g}(G)\left(\cos d_{g}(G)\right)^{-1}
\end{aligned}
$$

We put

$$
d_{1}=\inf \left\{t>0 ; \frac{\pi}{2} \leqq t(\cos t)^{-3}\left(1+\sin ^{2} t\right)^{1 / 2}\right\} .
$$

Then

$$
\frac{\pi}{2}>d_{1}>0.66 \quad \text { and } \quad d_{g}(G) \geqq d_{1} . \quad \text { Q.E.D. }
$$

\section{References}

[1] Boothby, W. M. and Wang, H.-C., On the finite subgroups of connected Lie groups, Comm. Math. Helv., 39 (1964), 281-294.

[2] Cheeger, J. and Ebin, D. G., Comparison Theorems in Riemannian Geometry, NorthHolland, Amsterdam, 1975.

[ 3 ] Sugahara, K., On the diameter of certain Riemannian manifolds, Publ. RIMS, Kyoto Univ., 11 (1976), 835-847. 
